
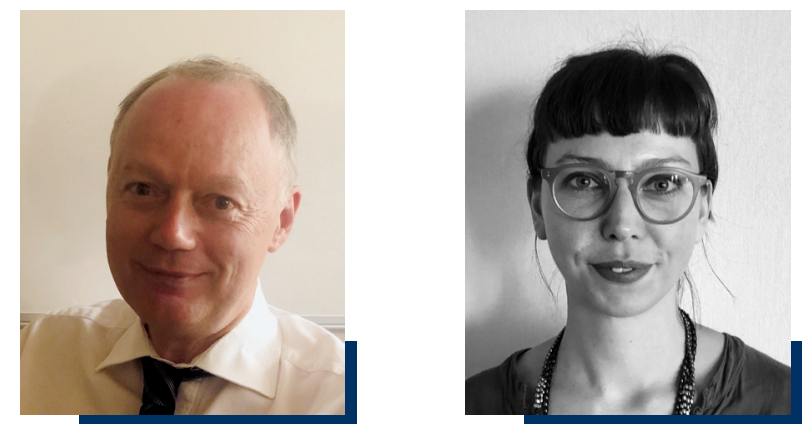

Gabor KESZLER

et Morgane LEQUÉRÉ-PLUCHON

Responsables budgétaires du CAS Pensions

au Service des retraites de l'État

\title{
Retour sur 10 ans de gestion budgétaire du Compte d'affectation spéciale Pensions
}

Mols-clés: finances sociales - gestion budgétaire - régime de retraites -
LOLF

La transformation du service des retraites de l'État en établissement public évoquée dans le projet de loi instituant un régime universel de retraite ${ }^{1}$ aura des implications sur le fonctionnement du compte d'affectation spéciale (CAS) Pensions. Un peu plus de dix ans après sa création, c'est l'occasion de faire le point et de revenir sur la gestion de ce véritable support financier du régime des retraites des fonctionnaires civils de l'État et des militaires.

Sur le projet de réforme des retraites et son incidence sur les fonctionnaires, voir les chroniques Repères d'actualité à la fin de chaque numéro de Gestion $\&$ finances Publiques et chaque mois sur www.gestion financespubliques.info. e CAS Pensions est une création typiquement lolfienne visant à rendre plus lisible le budget de l'État, en l'occurrence celui consacré aux pensions des fonctionnaires civils de l'État, des magistrats et des militaires. D'après I'article 19 de la LOLF, un compte d'affectation spéciale (CAS) a pour but de retracer des opérations budgétaires formant un ensemble cohérent et de mettre en face des dépenses des recettes particulières qui ont une « relation directe, par nature » avec les dépenses concernées. Mis en place en 2006, le CAS Pensions apporte une présentation budgétaire de l'ensemble des régimes de retraite et d'invalidité dont l'État a la charge : pensions de retraite et d'invalidité des fonctionnaires civils de l'État et des ouvriers d'État, pensions de retraite des militaires, pensions militaires d'invalidité, autres allocations assimilées. Le financement de ces dépenses figure ainsi dans une enveloppe budgétaire dédiée, hors budget général, votée en loi de finances. 
Le CAS Pensions constitue une mission budgétaire propre et comporte trois sections, retranscrites pour leur partie dépense par trois programmes budgétaires. Dans la LFI 2020, les dépenses du CAS Pensions s'élèvent à 60 milliards d'euros. Le programme 741 retrace le régime de retraite et d'invalidité des militaires et des titulaires de la fonction publique de l'État et constitue l'essentiel (56,1 Md€) des dépenses du CAS. Le programme 742 retrace le régime de retraite des ouvriers des établissements industriels de l'État (1,9 Md€). Enfin, le programme 743 est un « programme-miroir » qui permet simplement de retracer des dépenses de retraite déjà inscrites dans le budget général : pensions militaires d'invalidité et retraite du combattant financées par le programme 169 (98\% du P743), retraite des sapeurs-pompiers professionnels de la fonction publique territoriale, médaille militaire et légion d'honneur, etc. (financés par les P216, P161, P129, P195 et P198). Le programme 743 est un relais : il reçoit des subventions du budget général pour porter la dépense finale (1,6 Md€). Cette lisibilité du budget consacré aux pensions des fonctionnaires de l'État et des militaires constitue une des grandes réussites de la mise en place du CAS Pensions

\section{L'obligation d'équilibre : une règle d'or pour un CAS}

Tout CAS doit fonctionner en équilibre : le solde budgétaire cumulé, défini comme la somme des recettes diminuée de la somme des dépenses depuis la création du compte, doit être excédentaire à tout instant. Cette obligation emporte une double conséquence : des recettes spécifiques doivent être affectées au financement du CAS, par exception au principe d'universalité budgétaire, et celles-ci doivent pouvoir être adaptées pour couvrir les dépenses.

Dans une démarche de responsabilisation des employeurs, la contribution employeur, comme la cotisation salariale, est imputée sur la masse salariale de chaque ministère et employeur public pour identifier le coût global du recrutement d'un fonctionnaire de son recrutement jusqu'à sa retraite. Elle est intégrée dans les crédits de personnel sur la ligne spécifique « contribution CAS Pensions » ou "T2 CAS » dans le but d'infléchir la politique de recrutement des ministères et des établissements publics. Cependant, cette approche reste incomplète puisque cette ligne n'est pas intégrée dans la cible de dépense de l'État.
La LOLF pose également deux interdictions aux CAS : I'impossibilité d'effectuer des versements au bénéfice du budget général et le fait qu'aucune dépense de personnel (T2) ne peut y être imputée. Les frais de gestion du régime des retraites de l'État sont ainsi supportés par le programme 156 de la DGFiP et non par le CAS Pensions. C'est une spécificité du régime des retraites de l'État qui le distingue du fonctionnement en « caisse » qui est la règle pour les autres régimes. Ainsi, les coûts de gestion du régime sont votés chaque année par le Parlement.

Les dépenses du CAS sont limitées aux crédits ouverts en loi de finances, augmentés du report des crédits non consommés les années précédentes et qui doivent permettre de couvrir les aléas de dépenses non prévues. Ces reports de crédits ne sont pas plafonnés, à la différence des programmes du budget général pour lesquels les reports ne peuvent dépasser $3 \%$ des crédits ouverts en LFI. Dans le respect des conditions prévues à l'article 21 de la LOLF, le montant des reports du CAS ne peut toutefois pas excéder le solde budgétaire cumulé du CAS Pensions (7,8 Md€ à fin 2019). En 2020, les reports de crédits s'élèvent à 1,3 Md€, soit 2,2\% des crédits LFI.

L'affectation des recettes aux dépenses, la possibilité de report des crédits sans plafond et le calcul d'un solde budgétaire cumulé constituent les principales spécificités de la gestion budgétaire du CAS Pensions.

\section{Le solde cumulé du CAS, instrument majeur de pilotage}

Le CAS Pensions est une structure budgétaire simple, traduction d'un régime de retraites fonctionnant par répartition. Deux mécanismes assurent la trésorerie nécessaire aux paiements. Chaque fin de mois, elle est alimentée en recettes par les cotisations pensions issues de la paye des fonctionnaires civils et de la solde militaire avant le paiement des pensions. Ce mécanisme est d'autant plus robuste que la fonction de paye est souvent centralisée et que les cotisations issues de la paye civile bénéficient d'un déversement automatique dans les recettes du CAS Pensions. En complément, afin de faire face aux aléas de gestion, le CAS Pensions a bénéficié à sa création, en 2006, d'une recette exceptionnelle de $1 \mathrm{Md} €$. Depuis, le solde budgétaire cumulé du CAS assure la fonction de "fonds de roulement " et l'équilibre du compte.

Le niveau du solde cumulé constaté en exécution peut être différent de celui prévu en budgétisation par des aléas en dépenses comme en 
recettes. D'une part, la masse des prestations est sensible aux choix comportementaux des agents en matière de départ en retraite ainsi qu'aux taux de revalorisation annuelle des pensions, en général liés à l'inflation. D'autre part, l'essentiel des recettes est constitué par des contributions des employeurs publics dont les assiettes ne peuvent pas être prévues parfaitement en loi de finances initiale. Le CAS a donc besoin d'une réserve couvrant les aléas de prévisions, en plus de la nécessaire réserve qu'implique le décalage temporel entre les décaissements et les encaissements. Le report de crédits de début d'année remplit cette fonction en premier rang avec des crédits déjà ouverts, ensuite le solde cumulé permet l'ouverture de crédits si besoin.

Le montant correspondant au solde budgétaire cumulé ne traduit pas une immobilisation de trésorerie sur un compte de l'État: il n'y a pas de «cagnotte ». II ne peut pas non plus être utilisé pour financer d'autres types de dépenses et n'ouvre pas droit à la consommation de crédits budgétaires supplémentaires. Il permet uniquement d'assurer à chaque instant que toutes les dépenses du CAS Pensions auront été financées par des recettes préalables en lien direct avec la dépense.

L'importance des montants liés aux retraites explique la participation directe de la direction du Budget dans le pilotage financier du CAS Pensions à côté du Service des retraites de l'État avec qui elle partage la responsabilité des programmes budgétaires.

Les programmes 741 et 743 du CAS Pensions sont placés sous la responsabilité du Service des retraites de l'État (SRE), service à compétence nationale de la DGFiP. Le programme 742 relève de la direction du Budget. Le responsable de programme définit les objectifs stratégiques du programme, garantit sa mise en œuvre opérationnelle et s'engage sur la réalisation des objectifs associés. En plus de la fonction de responsable de programme, le SRE a une fonction d'ordonnateur, bien que l'essentiel des opérations budgétaires soit réalisé par le réseau comptable de la DGFiP selon les procédures simplifiées de dépenses sans ordonnancement pour les pensions et de recettes au comptant pour les cotisations.

En collaboration étroite avec les autres services de la DGFiP et la direction du Budget, le SRE joue le rôle d'opérateur du régime de retraite de la fonction publique d'État avec un fonctionnement de plus en plus proche de celui d'une caisse de retraite : financement par des cotisations salariales et employeur, un fonds de roulement, une documentation budgétaire annuelle (PAP, RAP, jaune budgétaire, NEB), une traduction comp- table dans le compte général de l'État et une activité de prévision développée qui se traduit par la diffusion de données d'intérêt général labellisées «statistiques publiques ». La qualité de prévision des dépenses de pensions est d'ailleurs un indicateur de performance du programme et se caractérise par des résultats très satisfaisants. Le SRE assure également l'animation du réseau payeur des pensions, pilote l'alimentation des comptes individuels retraite par les ministères et met en œuvre un " parcours usager » moderne auprès des agents en activité et des pensionnés via son portail numérique ENSAP. II n'assure pas encore de fonction comptable, restée à ce stade mutualisée au sein du réseau DGFiP.

\section{Les différentes réformes des retraites ont contribué à la maîtrise des dé- penses du CAS Pensions}

Les dépenses de pensions évoluent en fonction du nombre de pensionnés, de leur âge de départ et de leur espérance de vie, de la pension moyenne à la liquidation et des revalorisations. Les dépenses du CAS Pensions ont progressé fortement jusqu'en 2013 : en euros courants, elles sont passées de 46,4 Md€ en 2007 à 55,6 Md€ en 2013. Elles ont continué à progresser depuis, mais à un rythme moindre, pour atteindre 59,0 Md€ en 2019.

Depuis 2003, les différentes réformes des retraites ont agi sur les principaux paramètres d'un régime par répartition, d'où le terme parfois utilisé de "réforme paramétrique »: durée d'assurance requise, âge d'ouverture des droits, taux de revalorisation des pensions, mise en place de système de décote/surcote afin d'assurer la viabilité du système de retraite à long terme.

Pour permettre un fonctionnement équilibré du CAS pensions dans ce contexte d'augmentation des dépenses, les taux de contribution employeur ont nettement progressé depuis la création du CAS Pensions, passant de 49,90\% pour les civils et 100,00 \% pour les militaires en 2006 à, respectivement, 74,28 \% et 126,07 \% depuis 2014. Ces taux sont stables depuis 5 ans. Actuellement, ils assurent largement l'équilibre puisque le CAS Pensions a dégagé un excédent de 1,3 Md€ en 2019. Conséquence : le solde cumulé est passé de 1,0 Md€ en 2013 à 7,9 Md€ fin 2019, soit l'équivalent d'1,3 mois de dépenses du CAS. L'excédent budgétaire annuel a commencé sa décrue en 2018 mais devrait rester positif encore quelques années. Ensuite, il pourra jouer un rôle d'amortissement entre la dynamique des dépenses de pensions, attendues en hausse, et celle des cotisations, liées à l'évolution des effec- 
tifs et à la politique salariale de la fonction publique d'État.

Le taux de cotisation salariale augmente chaque année depuis 2010 dans le cadre de la convergence progressive du taux de retenue pour pension des fonctionnaires vers celui en vigueur pour le régime général. De 7,85 \% en 2010, le taux de cotisation s'établit à 11,10\% en 2020 .

La ponctualité du versement des cotisations et des contributions par les employeurs de fonctionnaires de l'État, de magistrats ou de militaires constitue un enjeu important pour le CAS Pensions dans la mesure où un recouvrement plus précoce permet de limiter le besoin de trésorerie de l'État lors du paiement des pensions. S'appuyant sur le réseau de la DGFiP, l'essentiel de ces versements est intégré dans les recettes du CAS Pensions de façon automatique par le biais de la paye État ou manuellement par un comptable spécialisé pour la solde des militaires, Orange ou la Poste. Le reliquat est versé par une grande diversité d'employeurs de fonctionnaires en détachement : établissements publics, collectivités locales, associations et entreprises.

Conformément aux préconisations de la Cour des comptes, des décrets prévoyant des majorations de retard pour le non versement dans les délais des contributions au CAS Pensions, ont été publiés fin 2018. Ils alignent chaque fois que possible, les obligations des employeurs sur celles existant au régime général ou à la $\mathrm{CN}$ RACL. Cette évolution rapproche le régime des retraites de l'État des règles de fonctionnement des autres régimes, organisés en caisse.

\section{Objectifs atteints pour le CAS Pensions. Et maintenant?}

Après plus de 10 ans de fonctionnement, les objectifs de lisibilité et de gestion financière équili- brée qui ont présidé à la création du CAS Pensions sont atteints. Les règles de gestion budgétaire et financière conçues et mises en place pour entrer dans l'univers de la LOLF ont pleinement démontré leur robustesse et leur adaptabilité à un environnement mouvant. Dans son analyse de l'exécution budgétaire 2018 du CAS Pensions, la Cour des comptes renouvelle ses observations positives concernant la soutenabilité de la mission Pensions, la régularité de l'exécution du CAS Pensions et la gestion du solde cumulé. Ces éléments confirment la maturité de la gestion budgétaire du CAS Pensions et sa situation financière maîtrisée.

Après avoir appelé à la mise en place d'une véritable caisse de retraite des fonctionnaires de l'État, " prolongation logique des réformes engagées depuis une dizaine d'années, avec la mise en place d'une part du CAS Pensions, qui permet une plus grande transparence des dépenses et des recettes, et d'autre part du $S R E$, en tant qu'administration chargée de la gestion », la Cour des comptes estime désormais que l'alignement projeté des différents régimes de retraite pourrait conduire à remettre en cause l'existence même du mode de gestion en régie des retraites des agents concernés par le CAS. Le service des retraites de l'État, acteur majeur du système des retraites en France, suit avec attention l'évolution des travaux parlementaires relatifs au projet de loi sur les retraites. En tout état de cause, la qualité de la gestion et du pilotage budgétaire, la capacité à mener la réforme de la gestion des retraites de l'État et à offrir de nouveaux services aux usagers sont autant d'atouts qui pourront être pris en compte lors de la refondation vers un système universel de retraites et dans la gestion sécurisée de la période de transition. 


\section{Pour aller plus loin}

Graphique : évolution des dépenses des trois programmes et du solde cumulé depuis 2006 (en M€)

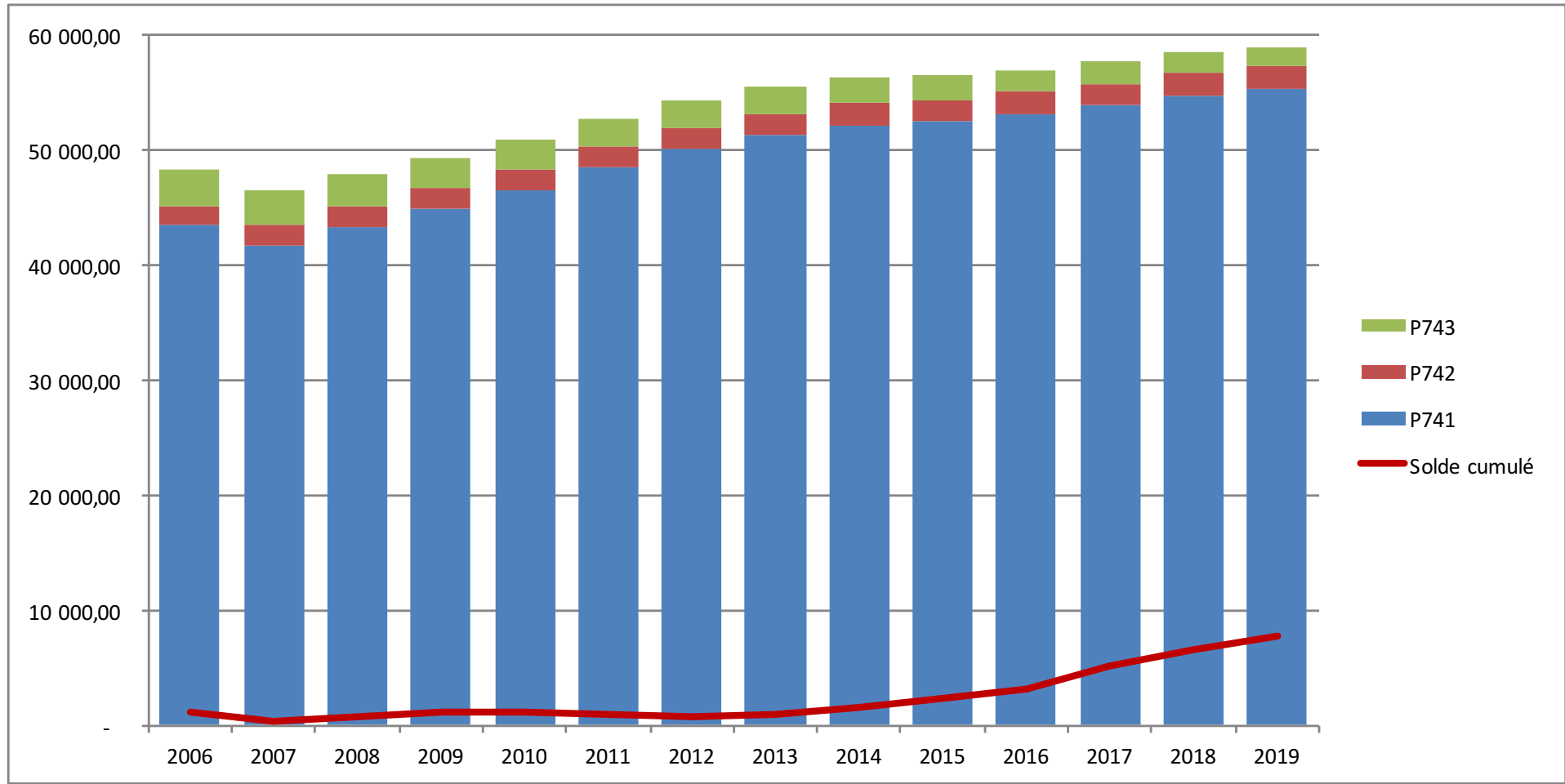

Source : DGFiP - Service des retraites de l'État

\section{Sites internet :}

https://retraitesdeletat.gouv.fr/ : rubrique «Professionnels» :

- Les données statistiques ;

- Le «CAS Pensions».

https://www.performance-publique.budget.gouv.fr/ :

- Guide de la LOLF

- Jaune «Pensions »

- Documents budgétaires : projet de loi de finances et documents annexés (PAP, annexes générales); projet de la loi de règlement et documents annexés (RAP, comptes de l'État, etc.) 